\title{
SIGNIFICADO DE LOS FACTORES SOCIALES Y CULTURALES EN EL DESARROLLO COGNITIVO
}

\author{
AdRIÁn Dongo M. ${ }^{1}$ \\ Universidad Estadual Paulista, Brasil \\ (RECIBIDO El 2/5/2009, ACEPTADO El 3/11/ 2009)
}

\begin{abstract}
RESUMEN
El problema del estudio de los factores sociales y culturales que intervienen en el desarrollo de la cognición se refiere a su tratamiento global y unilateral en relación con los factores individuales. Particularmente, el problema se manifiesta cuando no se distingue el factor de las interacciones sociales del factor de la transmisión cultural. En este trabajo analizaremos la acción de los factores sociales y culturales en el desarrollo de las estructuras del conocimiento, objetivando evidenciar sus acciones diferenciadas y solidarias. En función de ese objetivo, examinaremos, primeramente, las características y acciones complementarias de los factores del desarrollo (individuales y sociales); a continuación, analizaremos investigaciones realizadas en diferentes medios sociales y culturales que muestran sus acciones diferenciadas y complementarias. Finalmente, trataremos de evidenciar la novedad y la importancia del tratamiento relacional de los factores sociales y culturales para la investigación básica y aplicada.
\end{abstract}

Palabras clave: Factores sociales del desarrollo, interacción interindividual, transmisión social e cultural, psicologia genética.

\section{ABSTRACT \\ MEANING OF SOCIAL AND CULTURAL FACTORS IN COGNITIVE DEVELOPMENT}

The problem of studying social and cultural factors which take part in the cognition development is related to the global and unilateral treatment of those factors in relation to the individual ones. Specifically, the problem appears when we do not differ the interindividual social factors action from the social factors of cultural transmission. In the present study, we will analyze the action of social and cultural factors in the development of knowledge structures, aiming at showing its differentiated and complementary actions. As a result of this objective, in the first place we will analyze the characteristics and complementary actions of the development factors in general (individual and social ones). Secondly, we will analyze researches accomplished in different social and cultural environments which make its differentiated and complementary actions evident. Finally, we will try to make evident

1 Profesor de la Universidad Estadual Paulista, Brasil. E-mail: dongomontoyaa@.com.br 
the novelty and importance of the relational treatment of social and cultural factors for the basic and applied research.

Keywords: Development social factors, interindividual interaction, social and cultural transmition, genetics psychology.

\section{PROBLEMÁTICA DEL ESTUDIO DE LOS FACTORES SOCIALES Y CULTURALES EN EL DESARROLLO COGNITIVO}

Los factores sociales y culturales tienen una importancia decisiva en la explicación de la evolución del pensamiento, sin embargo, es necesario afirmar que ellos no actúan en bloque ni de modo unilateral e independientemente de los factores biológicos e individuales. Por tanto, es necesario diferenciar su accionar, pues, corrientemente, se le reduce a una única dimensión: la transmisión social y cultural.

No basta afirmar que el desarrollo del conocimiento en el individuo exige la participación de factores biológicos y sociales. Todas las teorías del desarrollo psicológico tienen en cuenta, de algún modo, ambos factores. Así, para las concepciones innatistas, los factores sociales no dejan de actuar en la actualización de estructuras hereditariamente programadas (Chomsky). Recíprocamente, para las concepciones ambientalistas los factores biológicos, no dejan de actuar, como tendencias o como principios de adquisición (asociación, refuerzos). Así, la consideración de factores tanto ambientales como biológicos no garantiza la superación de los reduccionismos y determinismos ambientalistas o biologistas.

Existen tres dificultades básicas en el estudio de los factores sociales y culturales: la primera, se refiere a la falta de diferenciación entre los factores biológicos e individuales por un lado, y los factores sociales por otro; la segunda se refiere al uso global e indiferenciado del término social. La tercera tiene que ver con los análisis dicotómicos y no relacionales de esos factores. Esas dificultades expresan una problemática amplia y de suma importancia que es necesario ser enfrentado tanto por la investigación básica como por la investigación aplicada.

Es común pensar como "factores sociales" los aspectos de transmisión educativa y de tradiciones culturales, que varían de una sociedad a otra. Es en esas presiones sociales colectivas que se piensa cuando se quiere explicar las variaciones cognitivas de una sociedad a otra, a comenzar por las diversas lenguas capaces de ejercer una acción importante sobre las propias operaciones y sobre sus contenidos. Sin embargo, esas presiones colectivas no son diferenciadas de los procesos colectivos más generales, como los factores de interacción o coordinación interindividual que atraviesan las diferentes culturas y sociedades. Ese tratamiento en bloque del factor social, sin la necesaria diferenciación de sus modos de acción, ha obstaculizado el análisis y la explicación más adecuada del desarrollo de la razón y de los conocimientos organizados en sistemas de composición.

La perspectiva global o indiferenciada se observa también en el análisis de los factores individuales, los cuales fueron reducidos a caracteres biológicos de preformación. Es necesario distinguir ciertos caracteres de funcionamiento adaptativo (y organizativo) que no son transmitidos en sentido estricto. Por otro lado, la teoría de la epigénesis (interacciones del genómio y del medio físico en el transcurso del crecimiento) ha 
contribuido enormemente para superar las concepciones dicotómicas del organismo y del medio. Los factores individuales, en ese contexto, tienen que ver sobre todo con los factores de coordinaciones internas o procesos de equilibración o autorregulación del individuo, que dependen tanto del funcionamiento adaptativo como de las coordinaciones interindividuales.

En este trabajo, analizaremos la acción de los factores sociales y culturales en el desarrollo de las estructuras del conocimiento mostrando sus acciones diferenciadas y complementarias en relación con los factores individuales.

Con ese objetivo examinaremos, en primer lugar, las características y acciones complementarias de los factores individuales y sociales del desarrollo, luego analizaremos las investigaciones realizadas en diferentes medios sociales y culturales que revelan acciones diferenciadas y complementarias de esos factores.

\section{DIFERENCIACIÓN Y SOLIDARIDAD DE LOS FACTORES DEL DESARROLLO COGNITIVO}

\section{Factores biológicos de epigénesis}

Son los factores relacionados a las interacciones del genomio con el medio físico en el transcurso del crecimiento, los cuales se manifiestan específicamente por la maduración del sistema nervioso. Según Piaget (1973), tales factores desempeñan un papel poco conocido, pero su importancia no es menos decisivo en el desarrollo de las funciones cognitivas. Esos factores no consisten en la acción de estructuras preformadas, sino, esencialmente, en abrir posibilidades nuevas para las conductas y estructuras anteriores. Los caracteres de la epigénesis se pueden encontrar en el desarrollo de las operaciones y estructuras lógico-matemáticas de la inteligencia en lo que se refiere a una cierta constancia o uniformidad sucesiva del desarrollo, sean cuales fueren las particularidades culturales y sociales en la que se forman los individuos. Así, inversiones en la sucesión de las fases de las nociones de conservación o modificaciones profundas en sus caracteres, de un medio a otro, probarían, al contrario, que los factores biológicos de las bases no intervienen en la evolución cognitiva de los individuos. Esa posibilidad deberá verificarse a través de investigaciones comparativas.

\section{Factores individuales de equilibración o de coordinación interna}

Si en el desarrollo de las operaciones interviniese solamente la acción continua del sistema epigenético (factor1), las fases no serían apenas secuenciales, pero también relacionadas a fechas cronológicas relativamente constantes, como la manifestación de la sexualidad en la pubertad. Las investigaciones interculturales muestran, por el contrario, progresos y atrasos muchas veces considerables conforme los medios sociales y culturales. Eso revela la existencia de otros factores además de los epigenéticos.

Los factores de equilibración se refieren a las acciones internas que se coordinan en sistemas cada vez más generales (esquemas). Las coordinaciones generales de los esquemas de acción suponen, entonces, sistemas múltiplos de autorregulación o equilibración, que dependerán de las circunstancias y de las potencialidades epigenéticas. Las propias operaciones de 
la inteligencia pueden ser consideradas formas superiores de esas regulaciones. Eso muestra, según Piaget (1973, 1975, 1976, 2002), al mismo tiempo, la importancia del factor de equilibración y su relativa independencia en lo que se refiere a preinformaciones biológicas.

Por otro lado, los factores de equilibración pueden ser concebidos como muy generales y relativamente independientes de transmisiones sociales y culturales particulares, sin embargo, estrechamente dependientes de coordinaciones interindividuales generales. Si las fases del desarrollo de las nociones de conservación se encontrasen en diferentes medios sociales y culturales, serían indicios del carácter general de las coordinaciones. Datos contrarios indicarían la marca de influencias culturales y educativas variables.

\section{Factores sociales de transmisión educativa y cultural}

Estos factores tienen que ver con las tradiciones culturales educativas que varían de una sociedad para otra. Es en esas presiones sociales diferenciadas que se piensa cuando se habla en "factores sociales". Es en esta forma de factor que se piensa cuando las competencias cognitivas varían de una sociedad a otra, particularmente de las diversas lenguas capaces de ejercer una acción importante tanto en la estructura de las propias operaciones como sobre el contenido de ellas.

Es evidente que este factor social podría ser solidario con las interacciones sociales, si las transmisiones educativas y culturales se produjeran en el contexto de verdaderos intercambios interindividuales (relaciones dialógicas, de cooperación, de respeto mutuo).

\section{Factores sociales de equilibración o de coordinación interindividual}

Las coordinaciones interindividuales se refieren a las interacciones sociales entre dos o más individuos, cuando se oponen, colaboran, discuten, entran en acuerdo, etc. Sus manifestaciones mismos restrictas y simples, se encuentran en todas las sociedades, independientemente de las particularidades culturales y educativas de una sociedad o de un medio social restricto.

En diferentes medios sociales y culturales, se observan ciertas conductas de intercambio entre niños y adultos, que actuan por su propio funcionamiento, independientemente de las transmisiones educativas. Así, en todos los medios los individuos se informan, colaboran, se oponen, discuten, etc. Y esa constante permuta interindividual interviene durante todo el desarrollo, según un proceso de socialización, que interesa tanto a la vida social de los niños entre sí, cuanto a sus relaciones con los adultos de todas las edades.

Así como Durkheim invocaba mecanismos sociales generales, sosteniendo que "sobre las civilizaciones existe la civilización”, asi también es indispensable, para tratar relaciones entre las funciones cognitivas y los factores sociales, comenzar oponiendo las coordinaciones generales de las acciones colectivas a las transmisiones culturales particulares que se cristalizan de manera diferente en cada sociedad. Es así que, en el caso en que se viese encontrar nuestros estadios y resultados en toda sociedad estudiada, eso en nada probaría que los desarrollos convergentes son de naturaleza estrictamente 
individual. Como es evidente que el niño cultiva, en todas partes, contactos sociales, desde la edad más tierna, eso mostraría cada vez más que existen ciertos procesos comunes de socialización que interfieren en los procesos de equilibración examinados anteriormente. (1973, p. 56)

Esa hipótesis es de fundamental importancia, pues recoloca en nuevas bases la relación entre individuo y sociedad en el desarrollo de la razón: entre ellos no existiría separación y dicotomía, mas si indisociabilidad y solidaridad.

... en el dominio de las funciones cognitivas, por lo menos, es bien posible que la coordinación general de acciones, cuya equilibración progresiva parece constitutiva de la formación de las operaciones lógicas o lógico-matemáticas, interesa tanto a las acciones colectivas o interindividuales cuanto a las acciones individuales. En otras palabras, quiera se trate de acciones ejecutadas individualmente o de acciones practicadas en común con permutas, colaboraciones, oposiciones, etc. se encontraran las mismas estructuras finales de operaciones o de cooperaciones, en cuanto co-operaciones; así, se podría considerarla lógica, en cuanto forma final de las equilibraciones, como simultáneamente individual y social: individual en cuanto general o común a todos los individuos, y también social, en cuanto general o común a todas las sociedades. $(1973$, p.57)

De ese modo, entre los factores individuales de coordinación intraindividual y los factores sociales de coordinación interindividual, existe una relación de solidaridad y dependencia cuando se analizan los procesos evolutivos del pensamiento conceptual. De ese modo, cuanto más una sociedad se pauta por coordinaciones interindividuales de carácter cooperativo, las posibilidades de que los individuos construyan coordinaciones intra-individuales de carácter operatorio serán más reales, y eso recíprocamente.

Sin embargo, conviene señalar que, a pesar de reconocerse la generalidad de ese factor, su acción concreta sobre el desarrollo del pensamiento dependerá del grado y calidad de las coordinaciones como tales. Existen interacciones que se restringen a actividades que objetivan el éxito práctico y no el intercambio regulado de puntos de vista. Existen también interacciones que se realizan en el contexto de la coacción y de la obediencia pasiva, y no del intercambio libre de pensamientos. Y, sabemos que los resultados de la interacción en el contexto de la coacción y de la obediencia pasiva serán opuestos a la interacción que se fundamenta en las leyes de la reciprocidad entre iguales y del respeto mutuo. Ni toda relación social se pauta por intercambios caracterizados por el diálogo, por el respeto mutuo y por la busca de objetividad.

\section{INVESTIGACIONES COMPARATIVAS SOBRE EL DESARROLLO DE LAS ESTRUC- TURAS COGNITIVAS}

Las investigaciones comparativas ponen en evidencia la necesidad de tener en consideración la acción diferenciada y, sin embargo, solidaria de los factores del desarrollo.

\section{Investigaciones referidas por Piaget}

Las investigaciones realizadas en Irán por Mohseni (1966), referidas por Piaget (1973), con niños alfabetizados de la ciudad de Teherán y niños analfabetos del campo, por medio 
de pruebas operatorias de conservación y test de desempeño, muestran los siguientes resultados: a) En relación con la sucesión de las nociones de conservación de substancia, de peso y de volumen, se encuentran, en líneas generales, en la misma sucesión de los estadios en la ciudad y el campo, en Irán y en Ginebra; b) Una diferencia sistemática de 2-3 años para las pruebas operatorias entre los jóvenes del campo y la ciudad, pero casi las mismas edades en la ciudad de Teherán y en Europa (1 a 2 años de atraso para los niños de Teherán); c)Atraso mas considerable para las pruebas de desempeño entre niños del campo y los de la ciudad, al punto que los primeros parecieran débiles mentales sin las pruebas operatorias.

La comprobación del orden secuencial de los estadios en Irán, como en otras latitudes, estaría mostrando la participación del factor biológico de la maduración. Sin embargo, para invocar con certeza ese factor sería necesario verificar no solo un orden secuencial de los estadios, pero aún de ciertas edades medias, cronológicamente fijas, de aparición. Los datos de Mohseni muestran un atraso sistemático de los niños del campo en relación a los niños de la ciudad. Eso estaría revelando la participación de otros factores, además de la maduración.

El segundo resultado claro de las investigaciones de Mohseni es el atraso bastante general de los niños del campo en relación con los de Teherán, en lo que se refiere tanto a las pruebas operatorias (conservaciones) cuanto de los test de desempeño. Como esta diferencia muestra, con clareza, la intervención de factores distintos de la simple maduración biológica, sería necesario aún distinguir cuales de los otros factores estarían interviniendo.

Como el propio autor de la investigación observó, en los niños del campo hay carencia sorprendente de actividades e interacciones. Eso podría estar indicándonos la presencia, al mismo tiempo, de un desarrollo frágil de las coordinaciones de las acciones individuales (factor 2), interindividuales (factor 4) y de transmisiones educativas (factor 3), una vez que los niños son analfabetos.

Para distinguir esos factores, el tercer resultado de Mohseni es instructivo. A pesar de la situación lamentable de los niños del campo, las reacciones de ellos a las pruebas operatorias son superiores a los resultados en los test de desempeño. El progreso significativo en relación con las pruebas de conservación parece indicar una diferencia de naturaleza entre coordinaciones bastante generales, necesarias para el funcionamiento de la inteligencia, y las adquisiciones especiales relativas a problemas particulares. Las pruebas operatorias darían lugar a mejores resultados porque son relacionadas a las coordinaciones necesarias y generales de la inteligencia (factor 2 y 4). Son necesarias en cuanto productos de la equilibración progresiva de las interacciones internas e externas, diferentemente de los desempeños que sufrirían atrasos importantes en función de factores culturales más especiales (factor 3).

Con relación a los factores sociales (3 y 4) es necesario aún examinarlos.

Para Piaget (1973), si las estructuras operatorias no se explicasen por las leyes de las coordinaciones más generales de la acción, sería necesario entonces pensar en factores más restrictos, de los cuales los dos principales podrían ser: primero, la acción educativa del adulto, análoga a la que producen los imperativos morales, y segundo, el lenguaje en cuanto cristalización de la sintaxis y de la semántica que, sobre formas generales, comportan una lógica. 
En la relación formativa de las coordinaciones por la educación del adulto, esa hipótesis es en parte verdadera, pues, estando el adulto más adelantado que el niño, puede él ayudarla y acelerar la evolución en el transcurso de los procesos educativos familiares y escolares. Pero, para Piaget, el problema es saber si ese factor es exclusivo, sobre todo cuando se trata de la acción educativa tradicional, como aquella defendida por Durkheim. Como se sabe, para este autor, la lógica emana, como a moral, de la estructura total de sociedad y se impone al individuo, gracias a las coacciones sociales, particularmente educativas.

Las investigaciones realizadas en Martinica por Boisclar, Laurendeau y Pinard, referidas por Piaget (1973), junto a una población de escolares, alfabetizados de acuerdo con el programa francés, muestran un atraso de cerca de 3 a 4 años en las principales pruebas operatorias. En este caso el atraso parece ser más bien imputable a los caracteres generales de la interacción social (factor 4 relacionado al factor 2) de lo que solamente a la carencia de las transmisiones educativas. O sea, una escolaridad común, como es el programa francés, no basta para asegurar un desarrollo normal de las estructuras operatorias. Además de eso, sería necesario, para evitar conclusiones prematuras, disociar las influencias familiares de las escolares.

Sobre el problema de la influencia del lenguaje en sus interacciones con el desarrollo operatorio, es importante llevar en cuenta las investigaciones de Sinclar (1967) sobre el desarrollo lingüístico del niño, y de Inhelder, Bovet e Sinclar $(1967,1977)$ sobre el papel del lenguaje en las experiencias de aprendizaje de las estructuras operatorias. Esas investigaciones revelan, por un lado, la estrecha relación entre operación y lenguaje y, por otra, que la operatividad es la que conduce a estructurar el lenguaje más de que lo inverso.

\section{Investigaciones realizadas en Brasil en niños que viven en barrios marginales (favelas)}

En 1983 fue realizada una investigación con niños de un barrio marginal (favela) de la ciudad de Sao Paulo (Dongo Montoya, 1996) con el propósito de explicar el desarrollo del pensamiento conceptual de esos niños. Para eso, en un primer momento, fue diagnosticado el nivel de organización de sus estructuras operatorias por medio de las pruebas operatorias clásicas en 120 niños de 7 a 12 años de edad. Los resultados de la investigación revelaron que para las nociones de conservación la gran mayoría de los niños (90\%), entre 7 y 8 años de edad, eran operatorias. Solamente alrededor de 11 y 12 años comenzaban a cambiar en la dirección del descubrimiento cualitativo de la substancia ( $71.5 \%$ en el nivel intermediario).

Esos datos muestran un atraso de 4 años en media en relación con los niños de Ginebra y también en relación a los niños de las clases medias de la ciudad de Sao Paulo.

Tabla N. ${ }^{\circ}$ 1. Conservación de la substancia.

\begin{tabular}{lccc}
\hline & \multicolumn{2}{c}{ Edades } & \\
Niveles & $\mathbf{7 - 8}$ & $\mathbf{9 - 1 0}$ & $\mathbf{1 1} \mathbf{- 1 2}$ \\
\hline No conservación & 90.0 & 56.0 & 19.0 \\
Intermedio & 7.5 & 28.0 & 71.5 \\
Conservación & 2.5 & 16.0 & 9.5 \\
\hline
\end{tabular}

Fuente: Dongo Montoya. 1996 p. 62, 63, 65. 
¿Cuál es el significado de esos datos para el problema de los factores del desarrollo cognitivo?

En primer lugar, a pesar del atraso del desarrollo, se constata que todos esos niños presentan regularidad secuencial en el niveles del desarrollo: en las diferentes pruebas operatorias no existe inversión o ausencia de construcción. Así, por la secuencia constatada, el factor epigenético no deja de estar presente en el desarrollo de esos niños. La acción de ese factor se ve reforzada por el hecho de haber observado en todos esos niños la organización previa de la inteligencia sensorio-motora o práctica.

El hecho del atraso constatado en la construcción del pensamiento operatorio revela que existirían otros factores en acción, principalmente los factores individuales de equilibración y los factores sociales de interacción. Pero, ¿cómo diferenciarlos?

Los resultados de la investigación muestran que, independientemente del nivel de escolaridad (niños escolarizados), la mayoría de los niños se encuentra en el nivel preoperatorio. Eso cuestionaba la acción exclusiva de la transmisión educativa y cultural en el desarrollo del pensamiento conceptual y apuntaba en la dirección de la importancia de otros factores, como aquella de la coordinación interindividual.

Para penetrar mejor en la acción del medio social, procedemos al estudio de la naturaleza de las interacciones con las personas que nos rodean (niños y adultos). Constatamos que en el plano de la acción práctica, esos niños interactuaban bastante con otras y que sus competencias prácticas eran muy desarrolladas. A pesar de eso, las interacciones en el plano de la representación se encontraban limitadas, pues ellas no sabían relatar las experiencias vividas, no justificaban sus acciones ni discutían con otros compañeros. Todo lo que sabían era salir bien en las tareas familiares y en los juegos motores y perceptivos.

El gran problema que se presentaba en esos niños era, entonces, la precariedad de los intercambios al nivel representativo y conceptual: nadie les exigía la reconstitución de las acciones realizadas por ellos mismos o la explicación y justificación de las acciones vividas cotidianamente. En otras palabras, esos niños interactuaban bien en el plano práctico, pero en el plano representativo y conceptual la interacción era bastante limitada. Se trataba de un ambiente donde vivían la "cultura del silencio", pues la marca de sus relaciones sociales era la opresión: "decir su palabra” y expresarse libremente podría tornarse algo contra ellos mismos.

Esa evidencia nos condujo a postular la hipótesis de que el factor social de coordinación interindividual en el plano de la actividad representativa y conceptual de los niños estudiados se encontraba limitada. O sea, en esos niños, a pesar de que las estructuras prácticas del conocimiento se encontraban desarrolladas, no se interiorizaban ni posibilitaban la organización conceptual del pensamiento por falta de intercambios simbólicos o semióticos adecuados. La situación se constituía, entonces, en un círculo vicioso: las limitaciones en la coordinación interindividual producían limitaciones en la coordinación intraindividual, y recíprocamente.

Del punto de vista de la educación familiar, la concepción de los padres estaba centrada en el suceso práctico del niño, en la obediencia ciega a las reglas establecidas pero no en la explicación y en la comprensión de las acciones. La escuela, por su parte, reforzaba 
ese tipo de relación, pues ella misma estaba centrada en la reproducción irreflexiva de las lecciones y saberes. Por tanto, la escuela producía una solidaridad negativa entre los factores de coordinación interindividual y de transmisión colectiva.

Creemos que esa es la situación básica de los niños marginados y oprimidos, presa además a una educación escolar opresiva y de pésima calidad.

Del punto de vista de las transmisiones educativas, se constata que la mayoría de los niños fueron alfabetizados en la misma lengua materna y con los mismos métodos educativos (centrados en la transmisión). Por tanto, la transmisión social y educativa no estaba contribuyendo en el desarrollo del pensamiento conceptual de esos niños. Para superar esa situación, era necesario otra forma de trabajo escolar, un trabajo que tenga en cuenta, efectivamente, el ejercicio de la coordinación interindividual (relaciones dialógicas y aprendizaje reflexivo).

Para reforzar y ampliar nuestra hipótesis realizamos una investigación de intervención (Dongo Montoya, 1996) que buscaba alterar la calidad de las coordinaciones interindividuales que conducirían a las coordinaciones intraindividuales.

En un grupo de 12 niños entre 8 y 10 años, de una misma serie escolar, que vivían en un barrio marginal de la ciudad Marília, localizada en el estado de Sao Paulo, iniciamos una investigación de intervención que buscaba posibilitar la construcción del pensamiento conceptual. Fueron diagnosticados los niveles de organización del pensamiento antes y después de la intervención, por medio de pruebas y situaciones preparadas para ese fin. Básicamente, la intervención consistió en inserir los niños en relaciones donde ellos podrían tener la oportunidad de planificar y evaluar actividades programadas conjuntamente y en acuerdo colectivo. Las actividades realizadas serían relatadas y reconstituidas en un orden espacio-temporal y causal, respetándose el estilo lingüístico de ellos. Tanto en los relatos como en los conflictos interpersonales, se buscaba tener en cuenta y respetar el punto de vista del interlocutor. Los medios de expresión serían los más diversos: desde los relatos verbales hasta las dramatizaciones y dibujos.

Después de un año de intervención en ese grupo de niños, los resultados de la evaluación de las competencias cognitivas evidenciaron que la organización conceptual del pensamiento fue alcanzado por la mayoría de los niños.

Los resultados revelarán que, desde el punto de vista de los factores del desarrollo, esos niños no presentaban ningún problema en relación al factor biológico de la epigénesis y que la enseñanza escolar no interfiere positivamente para el desarrollo de sus estructuras de conocimiento. Serían, por tanto, las limitaciones de las coordinaciones interindividuales en el plano de la representación conceptual que estarían interfiriendo negativamente en los procesos de equilibración o de coordinación intraindividual. Eso muestra evidentemente la relación solidaria y de dependencia entre el factor 2 y 4 .

Con eso, no queremos afirmar que toda escuela o todo proceso de alfabetización no puede hacer nada para contribuir en el proceso constructivo del pensamiento de esos niños. Por el contrario, su acción podría ser de gran relevancia si su postura autoritaria y bancaria cambiase en la dirección emancipadora y de una acción creadora. 
Es posible que exista una solidaridad positiva entre el factor de las interacciones interindividuales y de las transmisiones culturales en la escuela, pero, sobre la condición de que esta practique sistemáticamente relaciones de cooperación, de diálogo y de respeto mutuo. Es claro que esa nueva realidad solamente será siempre y cuando sean superados viejos modelos educativos y culturales reducidos a la simple misión de transmitir contenidos sin tener en cuenta la actividad reflexiva de los sujetos del conocimiento, los cuales sofocan la libertad y la creación de los individuos.

\section{CONCLUSIONES}

En el análisis de los factores del desarrollo del pensamiento conceptual, es necesario diferenciar las relaciones solidarias entre los factores sociales y los factores individuales. Para eso es importante distinguir, por un lado, los factores individuales de carácter epigenético y de equilibración (coordinación intraindividual) y, por otro, los factores sociales de transmisión cultural y de coordinación interindividual.

Las investigaciones interculturales evidencian un hecho primordial: la relación indisociable e irreductible entre el factor de coordinación intraindividual (equilibración individual) y la coordinación interindividual (equilibración social). El desarrollo de las coordinaciones interindividuales solicita el desarrollo de las coordinaciones intraindividuales, y eso recíprocamente; contrariamente, los déficits en las coordinaciones interindividuales propician déficits en las coordinaciones intraindividuales. Esa relación, como verificamos en los niños de barrios marginados, se muestra decisiva en el plano de la actividad representativa y conceptual: el déficit en el "intercambio simbólico" interindividual genera el déficit en las coordinaciones de los esquemas conceptuales.

Las investigaciones comparativas evidencian también que las transmisiones educativas y culturales, por sí mismas, no garantizan el desarrollo del pensamiento conceptual, pues los niños pueden ser alfabetizados pero eso no posibilita la construcción de las operaciones mentales. Sin embargo, esa aparente independencia no niega la posibilidad de solidaridad positiva entre la transmisión y las interacciones, pues un proyecto de enseñanza que propicie la creación y la recreación reflexiva de contenidos culturales, aprendizajes significativos e intercambios entre miembros de una comunidad de investigadores, puede, con seguridad, desarrollar las coordinaciones interindividuales y, por consecuencia, coordinaciones intra-individuales. Las experiencias escolares donde se estimuló el aprendizaje activo y reflexivo de los niños, así como el trabajo en grupo, son los mayores testigos de ese proceso solidario (Celin Freinet).

Considerar de manera diferenciada y solidaria los factores del desarrollo, destacadamente de los factores sociales, contribuye mucho para la investigación básica y para la investigación aplicada de los procesos constructivos del conocimiento. Así, la diferenciación de esos factores contribuye para un análisis más profundo de las realidades históricas y sociales envueltas en esos procesos, sobre todo, para alcanzar un modo nuevo de ver las realidades corrientemente aceptadas y tornar evidente aspectos ocultos pero de transcendencia fundamental.

Así, la potencia del sistema teórico de Piaget nos sorprende en el terreno de los procesos sociales y culturales envueltos en el desarrollo de la cognición humana. Pero, eso solamente 
ocurre cuando superamos viejos modelos interpretativos y conseguimos penetrar en el análisis más fino y más relacional (dialéctico) de los procesos y mecanismos envueltos en la acción individual y social.

\section{REFERENCIAS BIBLIOGRÁFICAS}

1. Dongo Montoya, A. (1996). Piaget e a criança favelada. Epistemologia genética: diagnóstico e soluções. Petrópolis: Vozes. Publicado en lengua española: Dongo Montoya, A. (2002). Piaget y los niños marginados. Epistemologia genética: Diagnóstico y soluciones. Lima: Pukio-URP.

2. Dongo Montoya, A. (1983). De que modo o meio social influi no desenvolvimento cognitivo da criança marginalizada: busca de uma explicação através da epistemologia genética de Jean Piaget. São Paulo - SP, Dissertação de mestrado, Universidade de São Paulo.

3. Durkheim, E. (1952). Educação e sociologia. São Paulo: Melhoramentos.

4. Piaget, J. (1973). Psicologia e epistemología genética. Por uma teoria do conhecimento. Rio de Janeiro: Forence.

5. Piaget, J. (1973b). Biologia de conhecimento. Petrópolis: Vozes.

6. Piaget, J. (1966). Necessité et signification des recherches comparatives en psychologie génétique. Journal International de Psychologie. Vol. 1.(1), 3-13.

7. Piaget, J. (1980). A Psicologia da criança. São Paulo: Difel.

8. Piaget, J. (1976). Le comportement, moteur de l'evolution. Paris: idées/Gallimard.

9. Piaget, J. (1975) L'équilibration des structures cognitives. Problème central du déveleppement. Paris: P.U.F.

10. Piaget, J. (2002). Seis estudos de Psicologia. Rio de Janeiro: Forence.

11. Sinclar, E. (1967). Langage et operations. Paris: Dunod.

12. Inhelder, B.; Bovet, M.; Sinclar, H. (1967). Développement et apprentissage. Rev. Suisse Psychol. Pure appl. 26, vol.1, 1-23.

13. Inhelder, B.; Bovet, M.; Sinclar, H. (1977). Aprendizagem e estruturas do conhecimento. São Paulo: Saraiva. 